\title{
Impaired generation of new subcategories and switching in a semantic verbal fluency test in older adults with mild cognitive impairment
}

\section{Laiss Bertola ${ }^{1,2 *}$, Maria Luiza Cunha Lima ${ }^{3}$, Marco A. Romano-Silva ${ }^{2,4}$, Edgar N. de Moraes ${ }^{5}$, Breno Satler Diniz ${ }^{1,2,4}$ and Leandro F. Malloy-Diniz ${ }^{1,2,4}$}

1 Laboratory of Clinical Neuroscience Investigations, Faculty of Medicine, Federal University of Minas Gerais, Belo Horizonte, Brazil

${ }^{2}$ National Institute of Science and Technology in Molecular Medicine, Faculty of Medicine, Federal University of Minas Gerais, Belo Horizonte, Brazil

${ }^{3}$ School of Linguistics, Federal University of Minas Gerais, Belo Horizonte, Brazil

${ }^{4}$ Mental Health Department, Faculty of Medicine, Federal University of Minas Gerais, Belo Horizonte, Brazil

${ }^{5}$ Medical Clinic Department, Faculty of Medicine, Federal University of Minas Gerais, Belo Horizonte, Brazil

\section{Edited by:}

Manuel Menéndez-González,

Hospital Álvarez-Buylla, Spain

\section{Reviewed by:}

Rosalux Falquez, University of Heidelberg, Germany

Esteban Hurtado, Universidad Diego

Portales, Chile

*Correspondence:

Laiss Bertola, Laboratory of Clinical Neuroscience Investigations,

Faculty of Medicine, Federal

University of Minas Gerais, Av.,

Alfredo Balena, 190 office 235, Belo

Horizonte, CEP 30.130-100, Brazil

e-mail: laissbertola@gmail.com
The semantic verbal fluency task is broadly used in the neuropsychological assessment of elderly subjects. Even some studies have identified differences in verbal fluency clustering and switching measures between subjects with normal aging and a clinical condition such as mild cognitive impairment $(\mathrm{MCl})$ and Alzheimer's disease, the results are not always consistent. This study aimed to compare clustering and switching measures of an animal's semantic verbal fluency task among normal controls (NC, $n=25$ ), amnestic mild cognitive impairment (aMCl; $n=25)$, amnestic multiple domain Mild Cognitive Impairment $(\mathrm{a}+\mathrm{mdMCl} ; n=25)$ and Alzheimer's disease (AD; $n=25)$ Brazilian subjects. The analyses were executed considering three (unifying the $\mathrm{MCl}$ subtypes) and four groups. As the data were not normally distributed, we carried out non-parametric tests (Kruskal-Wallis and Mann-Whitney tests) to evaluate the differences in performance in the measures of the verbal fluency test among the groups. The comparison demonstrated that the groups differed in the total of correct words produced, number of clusters and switching but the measure of new subcategories was the only with significant difference among the NC and all the clinical groups. The measure of new subcategories is the number of original subcategories inside the higher category of animals that the subject produced, such as farm, domestic, African animals. Our results indicate that semantic memory impairment is a visible and recent deficit that occurs even in non-demented subjects with very $\mathrm{MCl}$ and the implications of these findings are discussed.

Keywords: semantic verbal fluency, clustering, switching, subcategories, mild cognitive impairment, Alzheimer's disease

\section{INTRODUCTION}

Mild cognitive impairment (MCI) is common among older adults with prevalence estimates ranging from 3 to $42 \%$ (Yesavage et al., 2002; Ward et al., 2012), and subjects with MCI have increased risk of developing dementia (Han et al., 2012). The gold standard for the identification of MCI usually relies on comprehensive neuropsychological assessment. Nonetheless, neuropsychological assessments are expensive, time-consuming, need highly trained professionals to do and, thus, may not be readily available in clinical practice. Cognitive screening tests are widely available, have low costs and need no specialized training for its correct administration and interpretation of results. Though being routinely used in clinical practice to evaluate subjects with cognitive complaints, they are not sensitive to identify mild cognitive deficits (Diniz et al., 2007). As a consequence many older adults may not be correctly identified as MCI delaying the diagnosis until they reach the threshold for dementia.
Semantic verbal fluency (SVF) tests are one of the most common cognitive screening used in clinical and research settings. This task requires the initialization of a specific verbal behavior, the search for specific verbal information, and self-monitoring to avoid mistakes and repetitions. Additionally, it requires the availability of a specific semantic knowledge (Unsworth et al., 2011). The traditional scoring system is the sum of correct words produced within one minute (Strauss et al., 2006). The scores on this task has a good sensitivity and specificity to discriminate older adults with no cognitive impairment from those with dementia (Radanovic et al., 2009). Despite not useful to differentiate MCI from AD patients (Radanovic et al., 2009), lower scores on SVF is a predictor of progression from MCI to AD (O'Dowd et al., 2004; Saxton et al., 2004; Amieva et al., 2005; Cottingham and Hawkins, 2009).

In addition to the traditional score, other information provided by the SVF can add valuable information about cognitive 
status of an individual. Clustering is the grouping of words belonging to the same subcategory and is a measure of semantic memory knowledge. Switching is the change of subcategory across all possibilities and measure the efficiency to retrieve the stored information (i.e., measure of executive functioning). Previous studies found that AD patients produced smaller clusters and switched less frequently from subcategories than normal controls (Troyer et al., 1998; Murphy et al., 2006; Fagundo et al., 2008). Recently, Price et al. (2012) showed that subjects with that amnestic MCI produced, smaller cluster sizes and fewer new subcategories when compared to controls, but the frequency of switches was not significantly different. Murphy et al. (2006) reported similar results, except that amnestic MCI subjects did not differ in the mean cluster size from the healthy subjects.

As SVF is commonly used in clinical practice and the analysis of other aspects of this task can add important information on the cognitive status of a patient, we aim to evaluate whether measures of clustering, switching, and number of subcategories can help to distinguish MCI and AD patients from healthy older adults with no cognitive decline. In addition, we aim to compare these measures between subgroups of MCI subjects. We hypothesize that patients with MCI and AD will show significant differences in these measures in comparison to healthy controls; and that there is gradual decline in these measures as the subjects progress from amnestic single domain MCI to amnestic multiple domain MCI, and finally to $\mathrm{AD}$.

\section{MATERIALS AND METHODS PARTICIPANTS AND ASSESSMENT}

One hundred older adults were included in this study. These participants were referred to a secondary outpatient geriatric unit for a comprehensive geriatric clinical assessment. All participants underwent a comprehensive clinical interview done by a geriatrician, and evaluated with a standardized neuropsychological protocol (de Paula et al., 2013). In brief, this protocol included the tests for the principal cognitive domains. General cognitive status (Mini Mental State Exam), executive functions (Frontal Assessment Battery, Letter Fluency of S and Digit Span), visioconstructional ability (Stick Design Test and Clock Drawing Test), episodic memory (Rey Auditory Verbal Learning Test), semantic memory [Naming Test (TN-LINC) and Category Verbal Fluency (Fruits)], and language (Token Test). The caregivers answered the General Activities of Daily Living Scale (GADL) that evaluates performance on activities of daily living (de Paula et al., 2014).

The neurocognitive status of each participant was adjudicated taking into account all information from the clinical and neuropsychological assessments. The participants were classified into three groups: normal controls $(n=25), \mathrm{MCI}(n=50)$, and mild $\mathrm{AD}(n=25)$. MCI was diagnosed according to the Mayo Clinic Criteria (Petersen et al., 2001); AD was diagnosed according to the NINCDS-ADRDA criteria (McKhann et al., 1984). The normal control (NC) group included older adults without cognitive complains and scores above $-1.5 \mathrm{SD}$ of the mean according to local norms, adjusted for age and educational level.

The MCI participants was further subdivided into two groups: amnestic single domain-aMCI $(n=25)$ and amnestic multiple domain-a+mdMCI $(n=25)$ (Petersen, 2004). Amnestic MCI is defined by significant memory impairment and normal performance on other cognitive domains, no evidence of impairment in activities of daily living and preserved global cognition (Petersen et al., 2001). Amnestic multiple domain MCI is defined by significant memory impairment and of one or more additional cognitive domains (e.g., executive function, language), no evidence of impairment in activities of daily living and preserved global cognition. The local Ethics Committee approved this study and all participants and their families gave written consent.

\section{VERBAL FLUENCY TEST}

All participants did the category verbal fluency test (animals). They were asked to say names of animals within 1 minute, and were advised to not repeat already spoken animals. All the words were recorded, including repetitions and errors (when other words that not animals were spoken).

\section{Standard scores}

The scoring procedure included the number of correct words, excluding number of errors (occurrence of words that do not refer to any animal) and number of repetitions (animals that were spoken more than once).

\section{Clustering and switching scores}

The scores for clustering and switching were obtained according to Troyer et al. (1997). Clusters were formulated according to the shared attributes between animals (e.g., farm animals, pet animals, zoo), or when an animal appeared alone. For example, the following animals compose one cluster: cow, pig, horse (farm animals). The cluster sizes were computed after a second word of the same subcategory if generated in sequence (cluster size $=$ total of animals in a given cluster -1 ). For example, cluster with two words receive a size score of 1 , with 3 words a size score of 2 . If the word appeared alone, this cluster receives a size score of 0 . The mean cluster size is the sum of all clusters sizes generated (including single words, repetitions, and errors) divided by the number of clusters. Switching is the number of changes in cluster generation during the task (Troyer et al., 1997). For example, a subject can say: cow, pig, horse (farm animals), whale, and fish (see animals). This subject produced one switching since he changed between the clusters of farm animals to a cluster of sea animals. We also included the measures of new subcategories (number of original subcategories produced, excluding reoccurrence of animals belonging to the same subcategory, but produced in a non-sequence way), as suggested by March and Pattison (2006). In the given example the subject produced two original subcategories. Other subject could saw: horse, cow, dog, cat, and pig. These second example shows that the subject first produced a cluster of farm animals (horse and cow), second a cluster of domestic animals (dog, cat), and third another cluster of farm animals (pig). In total these subject produces only two new subcategories of animals once he produced twice clusters of farm animals.

Additionally we proposed the scoring of the number of effective clusters that were developed, in which the subject produced more than one exemplar for that subcategory (horse and cow, instead of only saying horse), and the mean size of these developed clusters. 


\section{STATISTICAL ANALYSIS}

We did Kolmogorov-Smirnov tests to evaluate the pattern distribution of the data. As the data were not normally distributed (data not shown), we carried out non-parametric tests (KruskalWallis and Mann-Whitney tests) to compare for differences in the performance in the neuropsychological tests and measures of the verbal fluency test between groups. We also calculated the corresponding effect size (r) for each comparison. Chi-square tests were performed to analyze differences in the distribution of dichotomous variables between the groups. Statistical significance was set according to the Holm-Bonferroni correction for multiple comparisons. All analyses were performed with the Statistical Package for Social Science (SPSS), v.21 for Windows (IBM Corp. Released, 2012).

We carried out the same set of analysis dividing the MCI group according MCI subtype (groups: normal control, aMCI, $\mathrm{a}+\mathrm{mdMCI}, \mathrm{AD})$.

\section{RESULTS}

\section{NC, MCI, AD}

The AD, MCI, and control participants did not differ in most demographic data, except that the normal control group showed a significant higher frequency of women according to the Chisquare test $\left(X^{2}=6.76, d f=2, p=0.009\right)$. Participants with $\mathrm{AD}$ had worse performance in all neuropsychological measures and activities of daily living (Table 1). Participants with MCI had intermediate performance between $\mathrm{AD}$ and controls (Table 1).

Table 2 shows the data from SVF variables. The groups significantly differed in total of words, number of clusters, new subcategories, and switching (Table 2).
Pairwise comparison using the Mann-Whitney nonparametric test showed that all the three groups differed from each other on the number of correct words produced (Table 2). The NC group produced more new subcategories than $\mathrm{MCI}$ and $\mathrm{AD}$, but there were no difference in these measure between the clinical groups $(p=0.072)$. The NC group performed significantly better than $\mathrm{AD}$ also for number of clusters and switching (Table 2).

\section{NC, aMCl, a+mdMCl, AD}

After we divided the MCI group in aMCI and a+MCI we found a significant difference between the groups in in daily living activities and general cognitive status (Table 3 ), and in the SVF measures of correct words, number of clusters, new subcategories, and switching (Table 4).

Pairwise comparison showed that the NC group significantly differed from all clinical groups in the number of new subcategories (Table 4). NC group performed significantly better than $\mathrm{a}+\mathrm{mdMCI}$ and $\mathrm{AD}$ subjects also in the SVF variables of correct words, number of clusters and switching. There were no significant differences between aMCI, a+mdMCI and AD for all SVF variables, except a difference between $\mathrm{MMCI}$ and $\mathrm{AD}$ in the number of correct words produced (Table 4).

The number of new subcategories is the only significant difference found between the NC and all the clinical groups, including the aMCI subtype (Figure 1). There were no differences between the four groups in the mean cluster size.

We found a marginal significant difference between the NC and $\mathrm{AD}$ groups in the number of developed clusters $(p=0.05)$ and the mean size of the developed clusters $(p=0.05)$.

Table 1 | Demographic description of NC, MCl, and AD groups.

\begin{tabular}{|c|c|c|c|c|c|c|c|c|}
\hline & \multicolumn{2}{|c|}{ NC } & \multicolumn{2}{|c|}{$\mathrm{MCl}$} & \multicolumn{2}{|c|}{$A D$} & \multicolumn{2}{|c|}{ K-W } \\
\hline Education & 4 & $(3-4)$ & 4 & $(2-4)$ & 4 & $(2.5-4)$ & 0.34 & 0.840 \\
\hline GADL & 26 & $(26-26)$ & 26 & $(25-26)$ & 20 & $(17.5-22)$ & 62.74 & $0.000^{\dagger}$ \\
\hline MMSE & 27 & (23.5-29) & 25 & $(23-27)$ & 20 & $(17-23.5)$ & 29.18 & $0.000^{\dagger \dagger}$ \\
\hline
\end{tabular}

$G A D L$, General Activities of Daily Living Scale; MMSE, Mini Mental State Exam. ${ }^{\dagger} \mathrm{NC}<\mathrm{MCl}<A D$; ${ }^{+} \mathrm{NC}>\mathrm{MCl}>A D$.

Table 2 | Verbal fluency measures description of $\mathrm{NC}, \mathrm{MCl}$, and $\mathrm{AD}$ groups.

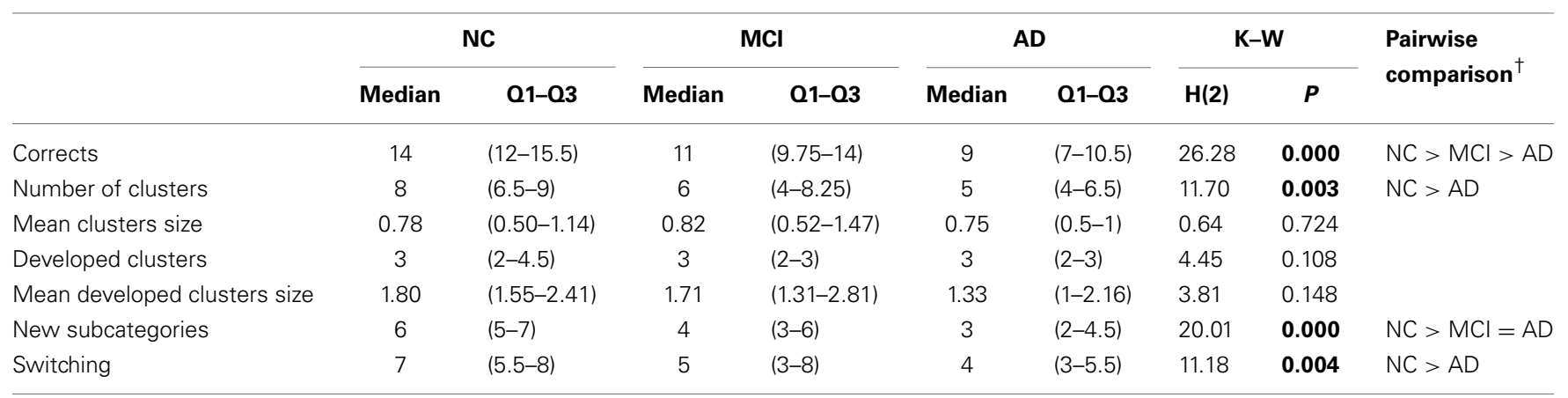

${ }^{\dagger}$ Mann-Whitney of verbal fluency measures between NC, MCl, and AD groups (significant p-value < 0.016 after Holm-Bonferroni correction for multiple analysis). Bold values of $p$ means that the $p$-value was statistically significant. 
Table 3 | Demographic description of NC, aMCl, a-mdMCl, and AD groups.

\begin{tabular}{|c|c|c|c|c|c|c|c|c|c|c|}
\hline & \multicolumn{2}{|c|}{ NC } & \multicolumn{2}{|c|}{ aMCl } & \multicolumn{2}{|c|}{$a+m d M C l$} & \multicolumn{2}{|c|}{$A D$} & \multicolumn{2}{|c|}{$\mathbf{K}-\mathbf{W}$} \\
\hline Education & 4 & $(3-4)$ & 4 & $(1.5-5)$ & 3 & $(2-4)$ & 4 & $(2.5-4)$ & 2.55 & 0.466 \\
\hline GADL & 26 & (26-26) & 26 & $(25-26)$ & 25 & $(24-26)$ & 20 & $(17.5-22)$ & 63.67 & $0.000^{\dagger}$ \\
\hline MMSE & 27 & $(23.5-29)$ & 26 & $(23-28)$ & 24 & $(22.5-26)$ & 20 & $(17-23.5)$ & 31.57 & $0.000^{\dagger \dagger}$ \\
\hline
\end{tabular}

$G A D L$, General Activities of Daily Living Scale; MMSE, Mini Mental State Examination. ${ }^{+} \mathrm{NC}<a \mathrm{MCl} ; a+m d M C l<A D ;+{ }^{+} \mathrm{NC}>a+m d M C l>A D ; a M C l>A D$.

Table 4 | Verbal fluency measures description of $\mathrm{NC}$, aMCl, a-mdMCl, and AD groups.

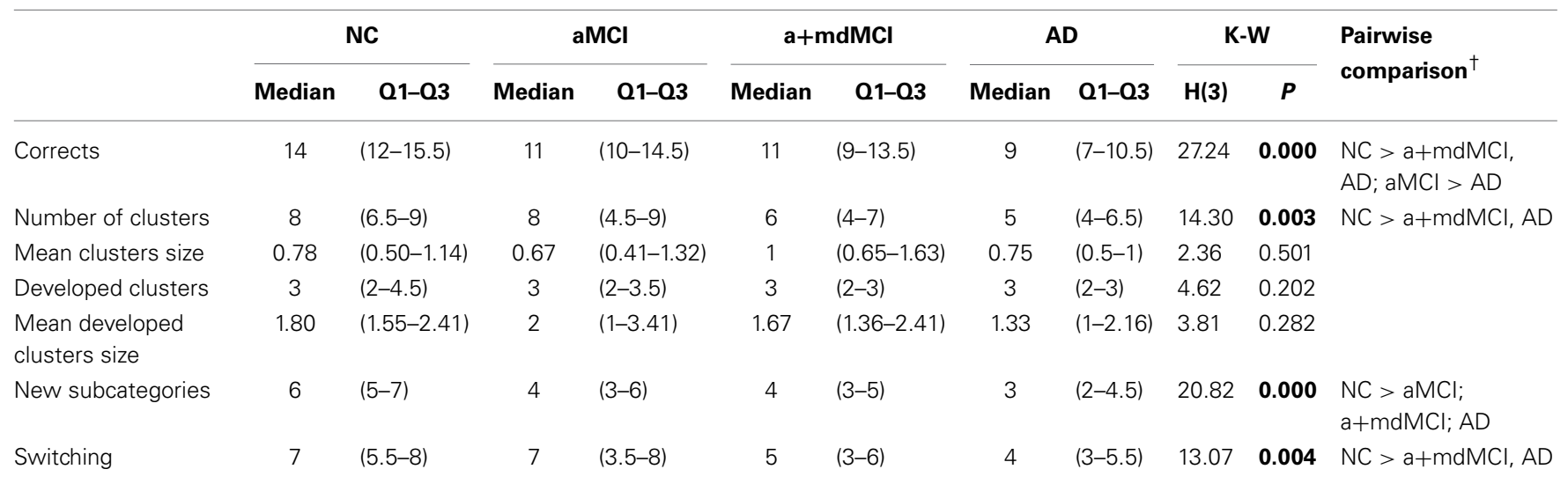

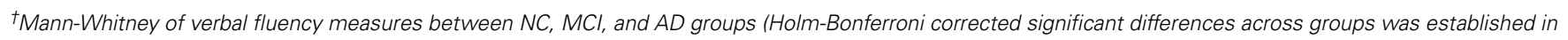
$p=0.008$ ). Bold values of $p$ means that the $p$-value was statistically significant.

\section{DISCUSSION}

The present study found that the number of animal subcategories produced in the SVF showed a significant decline in the amnestic MCI group compared to healthy controls. However, we did not observe significant differences in these measures between amnestic MCI, amnestic multiple-domain MCI and $\mathrm{AD}$ subjects. This may suggest that impairments in semantic memory are present in the early stages of the continuum of healthy cognitive aging, MCI, and dementia. In addition, we found that the number of clusters and switching were significantly reduced only in the amnestic multiple-domain and $\mathrm{AD}$ groups. These findings suggest that the latter subjects present with progressive difficulty to access the bulk of knowledge stored in semantic memory, possibly reflecting the presence of executive dysfunction in these subjects. Overall, these findings highlight the relevance of the assessment of other variables that can be extracted from an SVF task to understand the pattern of cognitive changes in the health cognitive aging, MCI and AD continuum.

The production of new subcategories was described as an alternative measure of semantic memory (March and Pattison, 2006). The generation of subcategories of animals depends on the knowledge of how a given animal relates to others or the shared attributes between them (e.g., pet animals or animals that are grown in farms) (Hoffman and Lambon Ralph, 2013). As animals in the same subcategory are closely related in the semantic memory system, once a person retrieves one animal it

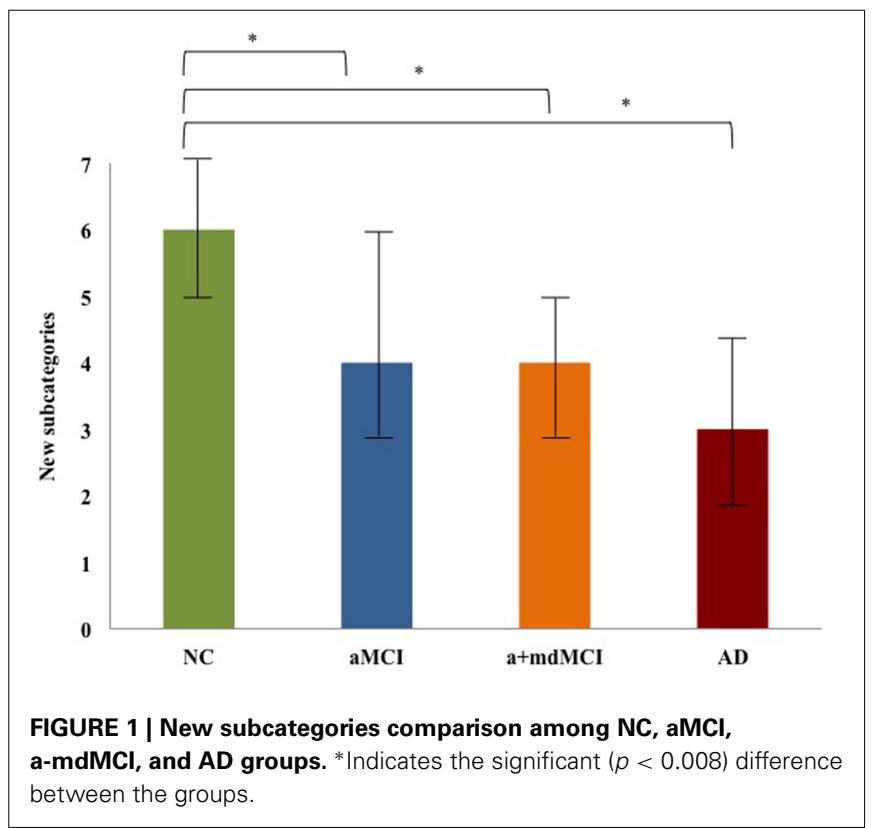

is easier to retrieve animals from that same subcategory following a structured semantic network (McClelland and Rogers, 2003). In our study we found that despite there were no significant differences in total of words produced in the SVF between controls and amnestic MCI participants, the latter showed a significant lower 
generation of animal subcategories. This finding suggests that the semantic memory system may be already disrupted in the earliest stages of the transition between normal cognitive aging and dementing disorders. Our findings are in line with the literature and highlight the importance of specifically evaluating semantic memory changes in addition to episodic memory in these subjects (Chan et al., 2001; Adlam et al., 2006; Joubert et al., 2008; Cuetos et al., 2009; Price et al., 2012).

It is worth noting that the switching measure were not significantly different between healthy controls and amnestic MCI participants, though were significantly lower in the amnestic multiple-domain MCI and AD. Switching is a measure of mental flexibility and indicates the ability to search and access novel information in the semantic memory system (Troyer et al., 1998; Nutter-Upham et al., 2008). These findings are in line with the theoretical decline in cognitive performance observed in the transition between amnestic MCI, multiple-domain MCI, and AD (Forlenza et al., 2009). Furthermore, our findings may suggest that the progressive decline in executive functions in subjects with MCI may indicate a higher risk of progression to AD (Rozzini et al., 2007).

The present results should be viewed in light of some limitations. We included a relatively small sample size what may have influenced the current analysis. Our sample has a low educational status. It is widely accepted that education is one of the main factors that influence the performance on a broad range of cognitive tests, including SVF (Radanovic et al., 2009). Also, education influences on how we store and retrieve information in the semantic memory (Reis and CastroCaldas, 1997; Mathuranath et al., 2003). Therefore, we cannot exclude the possibility that some of the results are biased due to the educational status of our sample. Furthermore, our control group has a significant difference of gender distribution, which may be a limitation for the study. Nonetheless, previous studies did not find a significant effect of gender in clustering and switching (Troyer and Moscovitch, 2006; Weiss et al., 2006). Therefore, additional studies, including greater sample sizes, including subjects with more years of education and with a prospective design, are necessary to evaluate whether SVF variables (i.e., generation of new subcategories, clustering, and switching) can help to differentiate between healthy controls, MCI and $\mathrm{AD}$ subjects, as well as to identify those subjects of progressing to dementia upon follow-up. Our results also present overlapping values of SVF measures across the groups. Future research should address whether these SVF attributes can complement the neuropsychological assessment to differentiate older adults with distinct levels of cognitive impairment.

Our study highlights the importance of evaluating other variables from the SVF tests, like the generation of new subcategories, cluster and switching, in subjects with MCI and AD. The impairment of production of new subcategories indicates the presence of semantic memory impairment in amnestic MCI subjects. Difficulties in SVF switching measures harbinger the presence of executive dysfunction and the diagnosis of multiple-domain MCI and AD. Finally, these measures may help to identify those subjects at a higher for dementia.

\section{ACKNOWLEDGMENTS}

This work was supported by the following grants: CBB-APQ00075-09, APQ-01972/12-10, APQ-02755-10, and APQ-0470610 from FAPEMIG; and 573646/2008-2 from CNPq. The funders had no role in study design, data collection, analysis, decision to publish, or preparation of the manuscript.

\section{REFERENCES}

Adlam, A.-L. R., Bozeat, S., Arnold, R., Watson, P., and Hodges, J. R. (2006). Semantic knowledge in mild cognitive impairment and mild Alzheimer's disease. Cortex 42, 675-684. doi: 10.1016/S0010-9452(08)70404-0

Amieva, H., Jacqmin-Gadda, H., Orgogozo, J.-M., Le Carret, N., Helmer, C., and Dartigues, J.-F. (2005). The 9 year cognitive decline before dementia of the Alzheimer type: a prospective population-based study. Brain 128, 1093-1101. doi: 10.1093/brain/awh451

Chan, A. S., Salmon, D. P., and La Pena, J. (2001). Abnormal semantic network for "Animals" but not "Tools" in patients with Alzheimer's disease. Cortex 37, 197-217. doi: 10.1016/s0010-9452(08)70568-9

Cottingham, M. E., and Hawkins, K. A. (2009). Verbal fluency deficits co-occur with memory deficits in geriatric patients at risk for dementia: implications for the concept of mild cognitive impairment. Behav. Neurol. 22, 73-79. doi: 10.3233/BEN-2009-0246

Cuetos, F., Rodriguez-Ferreiro, J., and Menendez, M. (2009). Semantic markers in the diagnosis of neurodegenerative dementias. Dement. Geriatr. Cogn. Disord. 28, 267-274. doi: 10.1159/000242438

de Paula, J. J., Bertola, L., Ávila, R. T., Assis, L. O., Albuquerque, M., Bicalho, M. A., et al. (2014). Development, validity, and reliability of the General Activities of Daily Living Scale: a multidimensional measure of activities of daily living for older people. Rev. Bras. Psiquiatr. 36, 143-152. doi: 10.1590/1516-4446-20121003

de Paula, J. J., Bertola, L., Ávila, R. T., Moreira, L., Coutinho G., Moraes, E. N., et al (2013). Clinical applicability and cutoff values for an unstructured neuropsychological assessment protocol for older adults with low formal education. PLoS ONE 8:e73167. doi: 10.1371/journal.pone.0073167

Diniz, B. S., Yassuda, M. S., Nunes, P. V., Radanovic, M., and Forlenza, O. V. (2007). Mini-mental State Examination performance in mild cognitive impairment subtypes. Int. Psychogeriatr. 19, 647-656. doi: 10.1590/S151644462008000400003

Fagundo, A. B., López, S., Romero, M., Guarch, J., Marcos, T., and Salamero, M. (2008). Clustering and switching in semantic fluency: predictors of the devolpment of Alzheimer's disease. Int. J. Geriatr. Psychiatry 23, 1007-1013. doi: 10.1002/gps.2025

Forlenza, O. V., Diniz, B. S., Nunes, P. V., Memória, C. M., Yassuda, M. S., and Gattaz, W. F. (2009). Diagnostic transitions in mild cognitive impairment subtypes. Int. Psychogeriatr. 21:1088. doi: 10.1017/s1041610209990792

Han, J. W., Kim, T. H., Lee, S. B., Park, J. H., Lee, J. J., Huh, Y., et al. (2012). Predictive validity and diagnostic stability of mild cognitive impairment subtypes. Alzheimers Dement. 8, 553-559. doi: 10.1016/j.jalz.2011.08.007

Hoffman, P., and Lambon Ralph, M. A. (2013). Shapes, scents and sounds: quantifying the full multi-sensory basis of conceptual knowledge. Neuropsychologia 51, 14-25. doi: 10.1016/j.neuropsychologia.2012.11.009

IBM Corp. Released. (2012). IBM SPSS Statistics for Windows, Version 21.0. Armonk, NY: IBM Corp.

Joubert, S., Felician, O., Barbeau, E. J., Didic, M., Poncet, M., and Ceccaldi, M. (2008). Patterns of semantic memory impairment in Mild Cognitive Impairment. Behav. Neurol. 19, 35-40. doi: 10.1155/2008/859657

March, E. G., and Pattison, P. (2006). Semantic verbal fluency in Alzheimer's disease: approaches beyond the traditional scoring system. J. Clin. Exp. Neuropsychol. 28, 549-566. doi: 10.1080/13803390590949502

Mathuranath, P. S., George, A., Cherian, P. J., Alexander, A. 1., Sarma, S. G., and Sarma, P. S. (2003). Effects of age, education and gender on verbal fluency. J. Clin. Exp. Neuropsychol. 25, 1057-1064. doi: 10.1076/jcen.25.8.1057.16736

McClelland, J. L., and Rogers, T. T. (2003). The parallel distributed processing approach to semantic cognition. Nat. Rev. Neurosci. 4, 310-322. doi: 10.1038/nrn 1076

McKhann, G., Drachman, D., Folstein, M., Katzman, R., Price, D., and Stadlan, E. M. (1984). Clinical diagnosis of Alzheimer's disease: report of the 
NINCDS-ADRDA work group under the auspices of department of health and human services task force on Alzheimer's. Neurology 34, 939-944. doi: 10.1212/WNL.34.7.939

Murphy, K. J., Rich, J. B., and Troyer, A. K. (2006). Verbal fluency patterns in amnestic mild cognitive impairment are characteristics of Alzheimer's type dementia. J. Int. Neuropsychol. Soc. 12, 570-574. doi: 10.10170\$13556177060 60590

Nutter-Upham, K. E., Saykin, A. J., Rabin, L. A., Roth, R. M., Wishart, H. A., Pare, N., et al. (2008). Verbal fluency performance in amnestic MCI and older adults with cognitive complaints. Arch. Clin. Neuropsychol. 23, 229-241. doi: 10.1016/j.acn.2008.01.005

O'Dowd, B., Chalk, J., and Zubicaray, G. (2004). Quantitative and qualitative impairments in semantic memory, but not phonetic fluency, as a potential risk factor for Alzheimer's disease. Brain Impairment 5, 177-186. doi: 10.1375/brim.5.2.177.58249

Petersen, R. C. (2004). Mild cognitive impairment as a diagnostic entity. J. Intern. Med. 256, 183-194. doi: 10.1111/j.1365-2796.2004.01388.x

Petersen, R. C., Doody, R., Kurz, A., Mohs, R. C., Morris, J. C., Rabins, P. V., et al. (2001). Current concepts in mild cognitive impairment. Arch. Neurol. 58:1985. doi: 10.1001/archneur.58.12.1985

Price, S. E., Kinsella, G. J., Ong, B., Storey, E., Mullaly, E., Phillips, M., et al. (2012). Semantic verbal fluency strategies in amnestic mild cognitive impairment. Neuropsychology 26, 490-497. doi: 10.1037/a0028567

Radanovic, M., Diniz, B. S., Mirandez, R. M., Novaretti, T. M. S., Flacks, M. K., Yassuda, M. S., et al. (2009). Verbal fluency in the detection of mild cognitive impairment and Alzheimer's disease among Brazilian portuguese speakers: the influence of education. Int. Psychogeriatr. 21, 1081-1087. doi: 10.1017/S1041610209990639

Reis, A., and Castro-Caldas, A. (1997). Illiteracy: a cause for biased cognitive development. J. Int. Neuropsychol. Soc. 3, 444-450.

Rozzini, L., Chilovi, B. V., Conti, M., Bertoletti, E., Delrio, I., Trabucchi, M., et al. (2007). Conversion of amnestic mild cognitive impairment to dementia of Alzheimer type is independent of memory deterioration. Int. J. Geriatr. Psychiatry 22, 1217-1222. doi: 10.1002/gps.1816

Saxton, J., Lopez, O. L., Ratcliff, G., Dulberg, C., Fried, L. P., Carlson, M. C., et al. (2004). Preclinical Alzheimer disease: neuropsychological test performance 1.5 to 8 years prior to onset. Neurology 63, 2341-2347. doi: $10.1017 /$ S1041610208007631

Strauss, E., Sherman, E. M. S., and Spreen, O. (2006). A Compendium of Neuropsychological Tests: Administration, Norms, and Commentary. Oxford, UK: Oxford University Press.
Troyer, A. K., and Moscovitch, M. (2006). "Cognitive processes of verbal fluency tasks," in The Quantified Process Approach to Neuropsychological Assessment, ed A. M. Poreh (New York, NY: Taylor and Francis), 143-160.

Troyer, A. K., Moscovitch, M., and Winocur, G. (1997). Clustering and Switching as two components of verbal fluency: evidence from younger and older healthy adults. Neuropsychol. Soc. 11, 138-146. doi: 10.1037/0894-4105.11.1.138

Troyer, A. K., Moscovitch, M., Winocur, G., Leach, L., and Freedman, M. (1998). Clustering and Switching on verbal fluency tests in Alzheimer's and Parkinson's disease. J. Int. Neuropsychol. Soc. 4, 137-143. doi: 10.1017/S1355617798001374

Unsworth, N., Spillers, G. J., and Brewer, G. A. (2011). Variation in verbal fluency: a latent variable analysis of clustering, switching, and overall performance. Q. J. Exp. Psychol. 64, 447-466. doi: 10.1080/17470218.2010.505292

Ward, A., Arrighi, H. M., Michels, S., and Cedarbaum, J. M. (2012). Mild cognitive impairment: disparity of incidence and prevalence estimates. Alzheimer's Dement. 8, 14-21. doi: 10.1016/j.jalz.2011.01.002

Weiss, E. M., Ragland, D., Brensinger, C. M., Bilker, W. B., Deisenhammer, A. A., and Delazer, M. (2006). Sex differences in clustering and switching in verbal fluency tasks. J. Int. Neuropsychol. Soc. 12, 502-509. doi: 10.10170S1355617706060656

Yesavage, J. A., O’Hara, R., Kraemer, H., Noda, A., Taylor, J. L., Ferris, S., et al. (2002). Modeling the prevalence and incidence of Alzheimer's disease and mild cognitive impairment. J. Psychiatr. Res. 36, 281-286. doi: 10.1016/S00223956(02)00020-1

Conflict of Interest Statement: The authors declare that the research was conducted in the absence of any commercial or financial relationships that could be construed as a potential conflict of interest.

Received: 17 March 2014; accepted: 12 June 2014; published online: 01 July 2014. Citation: Bertola L, Cunha Lima ML, Romano-Silva MA, de Moraes EN, Diniz BS and Malloy-Diniz LF (2014) Impaired generation of new subcategories and switching in a semantic verbal fluency test in older adults with mild cognitive impairment. Front. Aging Neurosci. 6:141. doi: 10.3389/fnagi.2014.00141

This article was submitted to the journal Frontiers in Aging Neuroscience.

Copyright (C) 2014 Bertola, Cunha Lima, Romano-Silva, de Moraes, Diniz and Malloy-Diniz. This is an open-access article distributed under the terms of the Creative Commons Attribution License (CC BY). The use, distribution or reproduction in other forums is permitted, provided the original author(s) or licensor are credited and that the original publication in this journal is cited, in accordance with accepted academic practice. No use, distribution or reproduction is permitted which does not comply with these terms. 